\title{
GBV Determinants of Survival of HIV-HAART Naive Patients to HAART Initiation at Bondo Sub-County Hospital: A Two Year Retrospective Cohort Study
}

\author{
Oghera Wesley Ooga ${ }^{1}$, Otundo Denis Orare ${ }^{1, *}$, Wambura Francis Muchiri ${ }^{2}$, \\ Chimbevo Mwagandi Lenny ${ }^{3}$, Kariuki James Ngumo ${ }^{4}$, Wang'ombe Anne ${ }^{1}$ \\ ${ }^{1}$ Institute of Tropical and Infectious Diseases, College of Health Sciences, University of Nairobi, Nairobi, Kenya \\ ${ }^{2}$ Institute of Topical Medicine and Infectious Diseases (ITROMID), College of Health Sciences, Jomo Kenyatta University of Agriculture \\ and Technology, Nairobi, Kenya \\ ${ }^{3}$ School of Health Sciences, Kirinyaga University, Kerugoya, Kenya \\ ${ }^{4}$ Center for Public Health Research (CPHR), Department of KEMRI, Nairobi, Kenya
}

Email address:

denotundo@yahoo.com (O. D. Orare)

${ }^{*}$ Corresponding author

To cite this article:

Oghera Wesley Ooga, Otundo Denis Orare, Wambura Francis Muchiri, Chimbevo Mwagandi Lenny, Kariuki James Ngumo, Wang'ombe Anne. GBV Determinants of Survival of HIV-HAART Naive Patients to HAART Initiation at Bondo Sub-County Hospital: A Two Year Retrospective Cohort Study. International Journal of HIV/AIDS Prevention, Education and Behavioural Science.

Vol. 3, No. 2, 2017, pp. 7-14. doi: 10.11648/j.ijhpebs.20170302.11

Received: February 10, 2017; Accepted: February 23, 2017; Published: May 10, 2017

\begin{abstract}
HIV-AIDS remains a major public health issue in Kenya with a prevalence of $5.3 \%$ and contributing to $29 \%$ of annual mortality. Despite marked improvement in the provision for care and treatment, a search for improvement of the current care and treatment programs may lead to better health outcomes. Various factors influence the prognosis of HIV disease. However, minimal research has been conducted to determine information gathered on enrollment influence HIV-AIDS prognosis. Early identification of how information can inform disease prognosis may aid in improving management strategies and increase quality of life of the HIV infected patients. This research aimed at determining how collected information influence times to HAART eligibility and factors influencing this duration. A retrospective cohort study was carried out in Bondo Sub County Hospital Comprehensive Care Centre. Primary data was collected from patient treatment files, in the period beginning $1^{\text {st }}$ January 2013 to $31^{\text {st }}$ December 2014 in data collection forms entered in Microsoft Excel, cleaned and transferred to Stata ver 13 for survival analysis. In the study period 2015 patients were enrolled and 164 (female $=93$, male $=71)$ satisfied the inclusion criteria. The medium survival time was 65 days. The WHO stage of enrollment $(p=0.0000)$ and age of enrollment $(\mathrm{p}=0.006)$ were found to be the major determinants of the time to HAART eligibility. The WHO stages of enrollment and age of enrollment were strongly associated with HIV prognosis and this could be attributed to level of immune status which is affected by both this factors. Age of enrollment and WHO stage of enrollment were the main variables captured in MOH 257 that inform on HIV-HAART naïve disease progression to HAART eligibility. This study needs to be done in a prospective study incorporating time dependent covariates so as to give a clear picture of the other covariates not picked in this study.
\end{abstract}

Keywords: Naïve Patients, Survival, HAART

\section{Introduction}

HIV is a lentivirus that causes HIV infection and leads to AIDs. The virus infects vital cells in the human immune system such as helper T-cells, specifically $\mathrm{CD}^{4} \mathrm{~T}$-cells leading to progressive failure of immune system allowing for life threatening opportunistic infections and cancers to thrive. The first case of HIV in Kenya diagnosed in 1984 and since then the epidemic has evolved to be a major public health concern [1]. Globally the WHO reports that 36.9 million 
people are living with HIV most of whom are found in sub Saharan Africa where Kenya is located [2]. According to NACC, 2014 the HIV adult prevalence in Kenya by the year 2014 stood at $5.3 \%$, adult being in reference to those fifteen years and above [4]. Siaya County has a population of 842,304 with a HIV prevalence of $23.7 \%$. Bondo sub county hospital is in Siaya County [1, 3]. In Kenya, HIV and AIDS remains a key public health problem, with a burden accounting for an estimated $29 \%$ of annual deaths, $20 \%$ maternal mortality and $15 \%$ of deaths of children under 5 years of age [5]. Marked improvement has been made in the fight of HIV-AIDS with the introduction of highly active antiretroviral therapy (HAART) leading to $80 \%$ coverage of people living with HIV requiring HAART despite this progress, HIV-AIDS still remains to be a major cause of mortality and morbidity in Kenya [1].

The points of entry in to care for HIV-AIDS management in the Kenya healthcare system include voluntary counseling and testing centers [5], prevention of mother to child testing points at the antenatal clinics, diagnostic testing and counseling centers in health care facilities at tuberculosis clinics and medical wards. The HIV-AIDS care program in Kenya takes care of enrolled patients in two categories. There are those who are enrolled on care and the others care and treatment [4]. The care and treatment patients are those who are enrolled on HAART and cotrimoxazole prophylaxis therapy. Those on care are mainly on cotrimoxazole prophylaxis therapy since they are yet to satisfy the criteria for HAART initiation. On being enrolled on HIV care the patient information is captured on a blue form known as $\mathrm{MOH} 257$ which captures the patient basic information and is registered into the pre-ART-register, $\mathrm{MOH}-316 \mathrm{~A}$ on subsequent visit the patient data is captured on this blue form. The information recorded on the blue card (MOH 257) includes: Patient profile or the bio data of the patient, Patient source, Patient art history, HIV status of family member, ART therapy and Visitation data.

The algorithm for adult enrollment onto care and treatment current in use to initiate on HAART [6] do not work for certain patient and other patient counseled and may be found to be eligible for HAART initiation. The algorithm also notes that at patient enrollment those who do not qualify for HAART initiation put on care and followed up until when they satisfy the initiation criteria. Although in the long run all those diagnosed with HIV end up being initiated on HAART, lack of understanding of factors that influence the transition into requirement for HAART may lead to non-provision of non-optimal care. An understanding of how these factors influence the deterioration of the patients' immune system leading to the need to initiate HAART may inform policy and help tailor the kind of care provision which would identify the risk factors leading for the need for HAART. Currently, HIV program in Kenya is not able to ascertain whether the data collected on the blue card has information that influence the transition of the patients enrolled on care to their transition into the need for them being enrolled on HAART. Therefore, development of algorithm that can be used to predict the entry and initiation point of HAART is crucial for management of HIV patients in Kenya.

\section{Methods}

Study site: This study was conducted at the Bondo Subcounty hospital comprehensive care center (CCC).

Study design: A retrospective cohort analysis of pre-art register on all HIV positive HAART naïve patients enrolled in Bondo sub-county comprehensive care clinic was conducted. A survival model of 2015 adult HAART naïve patients receiving all the other aspect of comprehensive care except HAART care only enrolled on at the Bondo sub County comprehensive care clinic were involved therefore sampling was not required but rather the period of study and all those enrolled was of essence. An inclusion exclusion criterion of new enrollment and the time to HAART initiation was applied to this total enrolled to get the numbers for the study and patients who by end of the study had not satisfied criterion for HAART initiation were right censored.

Data collection: Primary data was collected from patient treatment files, in the period beginning $1^{\text {st }}$ January 2013 to $31^{\text {st }}$ December 2014 using data collection forms, entered into Microsoft Excel, cleaned and transferred to Stata 13 for survival analysis.

Data analysis: Data was analyzed for Survival analysis [7]; survival function $\mathrm{S}(\mathrm{t})$ and hazard function $\mathrm{h}(\mathrm{t})$ [8] and survival curves were generated. In view that some patients were lost in the follow up, the Kaplan Meier product limit method of estimating survivorship function was employed [9]. The log rank test was used to statistically compare two or more groups within a single fixed covariate variable [10]. The Cox proportional hazard model was developed and the covariates tested included marital status, gender, patient source and how they affected the rate to HAART initiation. A model analysis and deviance was done as a test for the overall statistical significance.

Ethical considerations: Ethical Clearance to carry out the research was obtained from Kenyatta National Hospital (KNH) University of Nairobi (UON) Ethics and Review Committee. Authorization to conduct the study at the Bondo Sub- County Hospital was given by the Medical Superintendent following perusal and approval by the research committee of the facility.

\section{Results}

\subsection{Demographic Factors}

At enrollment, the patients were assessed and only 164 qualified for classification as per World Health Organization (WHO 2014) guidelines. Among these, 78 were classified as WHO stage I and 86 were classified as WHO stage II. The mean age of enrollment during the study period was 36.78 with a standard deviation of 12.8 . The means $95 \%$ confidence interval was $[34.8,38.75]$. The gender distribution at enrollment was male $43 \%(n=164)$ while the 
female were $57 \%$. All the observed patients during the period were eventually eligible for enrolled into HAART at study end time of December 2014. At enrollment, 90 patients were in a monogamous marriage which were the majority, 29 in a polygamous marriage, 5 divorced, 2 cohabiting with their partners, 12 widowed and 26 of them were single.

Table 1. Demographic Profile of Respondents.

\begin{tabular}{lll}
\hline Characteristics & Count (\%) N=164 & $\mathbf{9 5 \% C . I}$ \\
\hline Marital Status & & \\
Cohabiting & $2(1.2 \%)$ & $0.22-3.80$ \\
Divorced & $5(3.0 \%)$ & $1.12-7.13$ \\
Married monogamous & $90(54.9 \%)$ & $47.24-62.30$ \\
single & $26(15.9 \%)$ & $11.0-22.27$ \\
widowed & $12(7.3 \%)$ & $4.12-12.47$ \\
Married polygamous & $29(17.7 \%)$ & $12.56-24.29$ \\
\hline
\end{tabular}

\begin{tabular}{lll}
\hline Characteristics & Count (\%) N=164 & $\mathbf{9 5 \% C . I ~}$ \\
\hline Gender & & \\
Male & $70(42.7 \%)$ & $36.50-49.11$ \\
Female & $94(57.3 \%)$ & $49.66-64.64$ \\
Patience source for enrolment & \\
IP/DTC & $99(60.4 \%)$ & $52.72-67.54$ \\
OPD/DTC & $8(4.9 \%)$ & $2.34-9.48$ \\
VCT & $57(34.8 \%)$ & $27.88-42.33$ \\
\hline
\end{tabular}

Patients coming to be enrolled were referred from the three entry points; in-patient diagnostic and testing point, outpatient diagnostic testing center and voluntary testing centers. Majority of the patients (99) were from outpatient diagnostic testing center, 57 from voluntary testing centers but a few from 8 in-patient diagnostic and testing point (Figure 1).

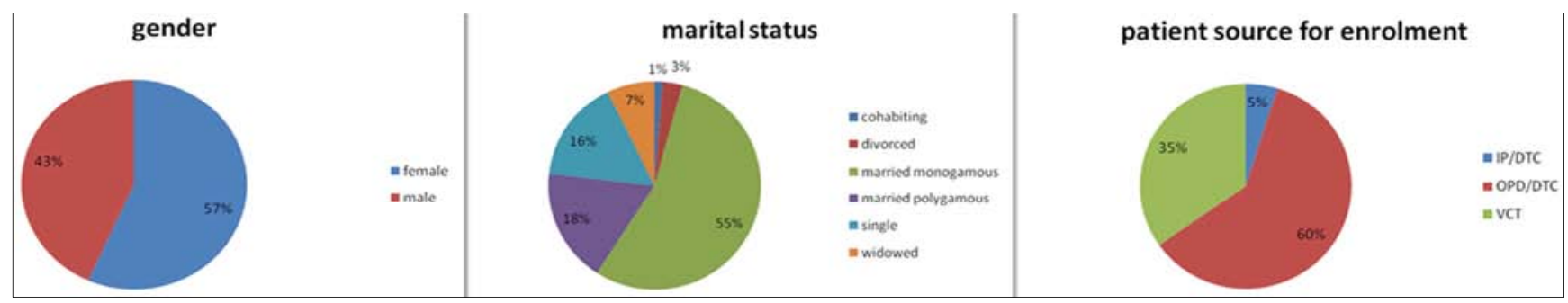

Figure 1. (a) Gender distribution at enrollment; (b) Marital status at enrollment; (c) Source of patient for enrolment to the care program.

The study also seemed to explore the knowledge of HIV status of family member as represented in Figure 2. On enrollment, the patient knowledge of a family member diagnosed with HIV showed that 107 of them had a known close family member with a confirmed HIV diagnosis while 57 had no knowledge of any family member with confirmed HIV status.

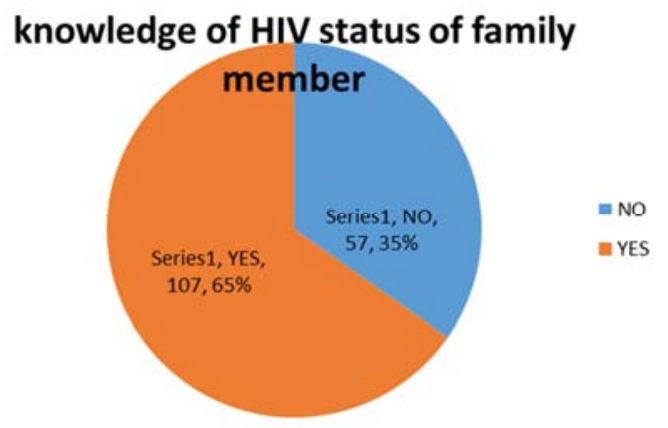

Figure 2. Knowledge of a family member diagnosed with HIV.

\subsection{HIV Positive HAART-Naive Patient's Survival}

A total of 164 patients were enrolled in the study and all at the end of the study had satisfied eligibility criteria for HAART initiation. The minimum exit time was 10 days, the median exit times was 65 days the maximum was 851 days with exit being satisfying eligibility to HAART. The $25^{\text {th }}$ percentile of survival time was 37 days and the $15^{\text {th }}$ percentile was 181 days.

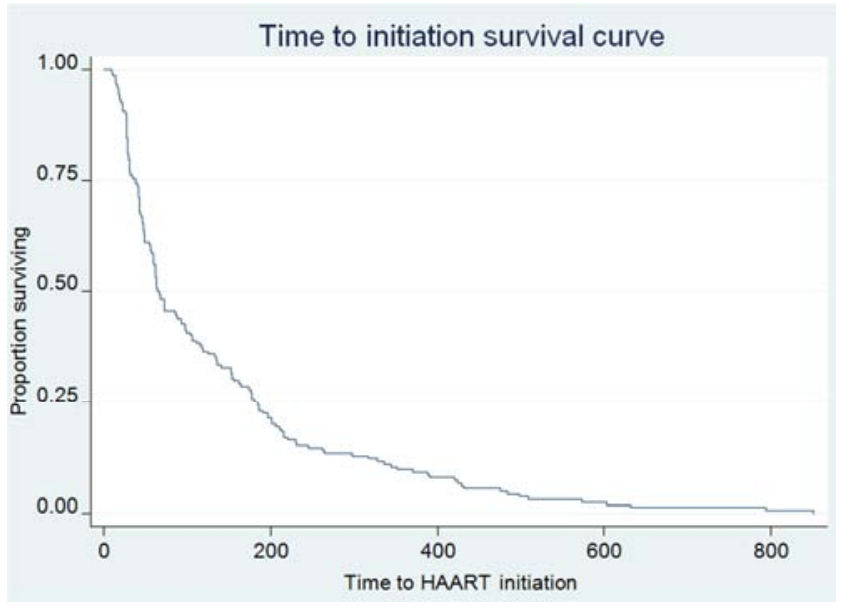

Figure 3. Patient survival curve for all enrolled patients.

\subsection{Non-Parametric Test for Categorical Variables}

Kaplan Meier survival curves were used to analyze survival and the Wilcoxon (Breslow) test for equality of survivor functions was used to ascertain if there was significant difference in the survival distribution of the categorical variable. This was because it was observed that with a median of 65days it implied that event of interest appeared 


\subsubsection{Analysis for Gender}

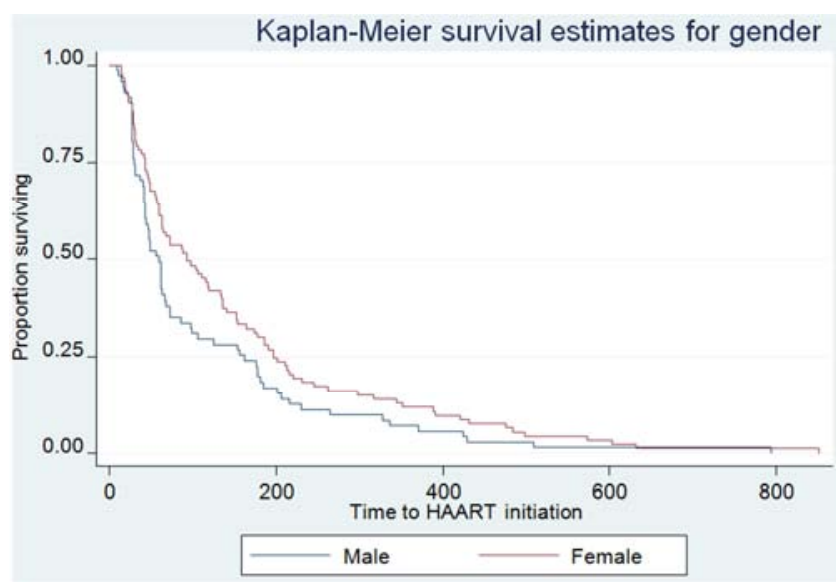

Figure 4. Kaplan Meier survival estimates for gender.

In this plot, it was observed that men generally met the time to HAART eligibility in early as compared to the females. This observation was then to be put to a statistical test to show if it was significant or just a chance occurrence by running the Wilcoxon (Breslow) test.

Table 2. Wilcoxon (Breslow) test for equality of survivor functions for gender.

\begin{tabular}{llll}
\hline Gender & Events observed & Events expected & Sum of ranks \\
Male & 71 & 59.54 & 1241 \\
Female & 93 & 104.46 & -1241 \\
Total & 164 & 164 & 0 \\
\hline
\end{tabular}

Chi2 $(1)=4.34$ p-value $=0.0275$

This was done to find out if gender played a role in determining the patient's progress to HAART eligibility criteria. It was statistically significant and this meant it would be included in the final model

\subsubsection{Analysis for Marital Status}

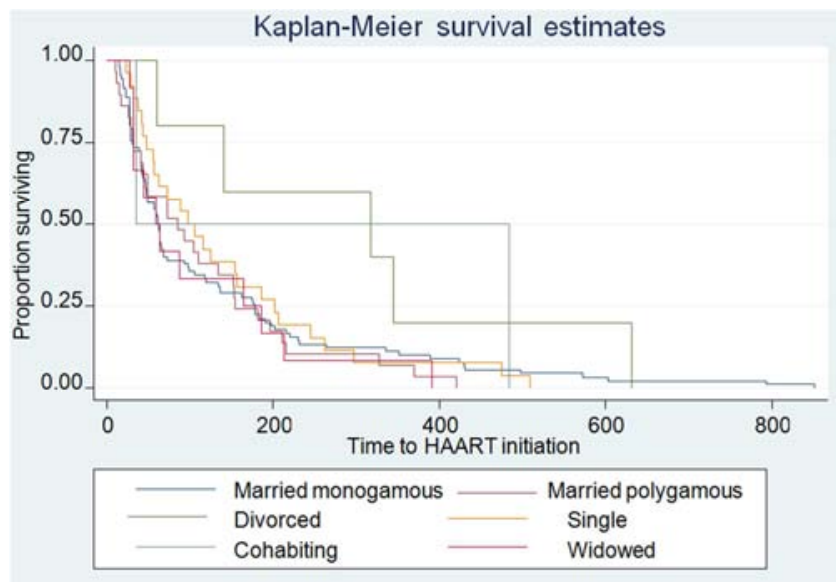

Figure 5. Kaplan Meier survival estimates for marital status.

In this plot, it was shown that none of the marital status produced observable difference in the plots showing time to
HAART eligibility. This observation was then to be put to a statistical test to show if indeed this was to be confirmed. This was done by running the Wilcoxon (Breslow) test.

Table 3. Wilcoxon (Breslow) test for equality of survivor functions for marital status.

\begin{tabular}{llll}
\hline Marital status & $\begin{array}{l}\text { Events } \\
\text { observed }\end{array}$ & $\begin{array}{l}\text { Events } \\
\text { expected }\end{array}$ & $\begin{array}{l}\text { Sum of } \\
\text { ranks }\end{array}$ \\
\hline Married monogamous & 90 & 86.85 & 840 \\
Married polygamous & 29 & 25.29 & 151 \\
Divorced & 5 & 10.15 & -444 \\
Single & 26 & 28.17 & -555 \\
Cohabiting & 2 & 3.35 & -64 \\
Widowed & 12 & 1021 & 72 \\
Total & 164 & 164 & 0 \\
\hline
\end{tabular}

Chi2 (5) $=5.69$ p-value $=0.338$

This was not statistically significant and would not be included in the final model.

\subsubsection{Analysis for Patient Source}

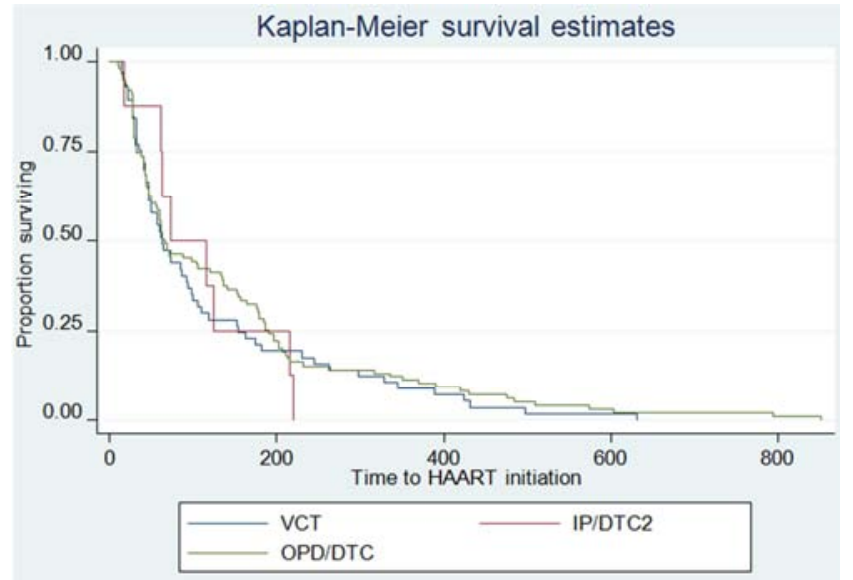

Figure 6. Kaplan Meier survival estimates for patient source.

In this plot, it was shown that none of the patient sources produced observable difference in the plots showing time to HAART eligibility event. This observation was then to be put to a statistical test to show if indeed this was to be confirmed. The Wilcoxon (Breslow) test was then run.

Table 4. Wilcoxon (Breslow) test for equality of survivor functions for patient source.

\begin{tabular}{llll}
\hline Patient source & Events observed & Events expected & Rank \\
\hline VCT & 57 & 52.58 & 336 \\
IP/DTC & 8 & 7.62 & -143 \\
OPD/DTC & 99 & 103.8 & -193 \\
TOTAL & 164 & 164 & 0 \\
\hline
\end{tabular}

Chi (2) $=0.52$ p-value $=0.7716$

This was not statistically significant and would not be included in the final model. 


\subsection{Analysis for WHO Stage at Enrollment}

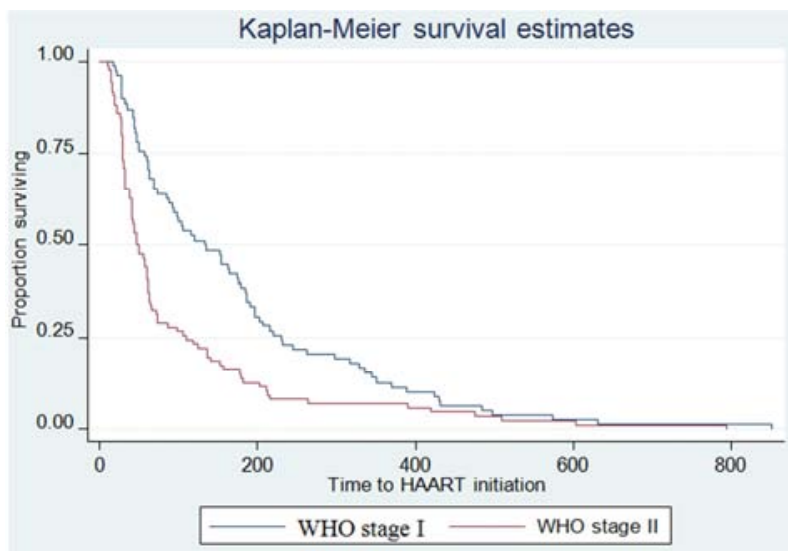

Figure 7. Kaplan Meier survival estimates for WHO stage at enrollment.

In this plot, it was observable that WHO stage II met the time to HAART eligibility early as compared to the WHO stage I. This observation was then to be put to a statistical test to show if it was significant or just a chance occurrence.

Table 5. Kaplan Meier survival estimates for WHO stage.

\begin{tabular}{llll}
\hline WHO stage & Events observed & Events expected & Rank \\
\hline Stage I & 78 & 100.44 & -2760 \\
Stage II & 86 & 63.5 & 2760 \\
Total & 164 & 164 & 0 \\
\hline
\end{tabular}

Chi2 (1) $=21.10$-value $=<0.0001$

This was statistically significant and would be included in the final model.

\subsection{Analysis for Knowledge of Diagnosed HIV + Family Member}

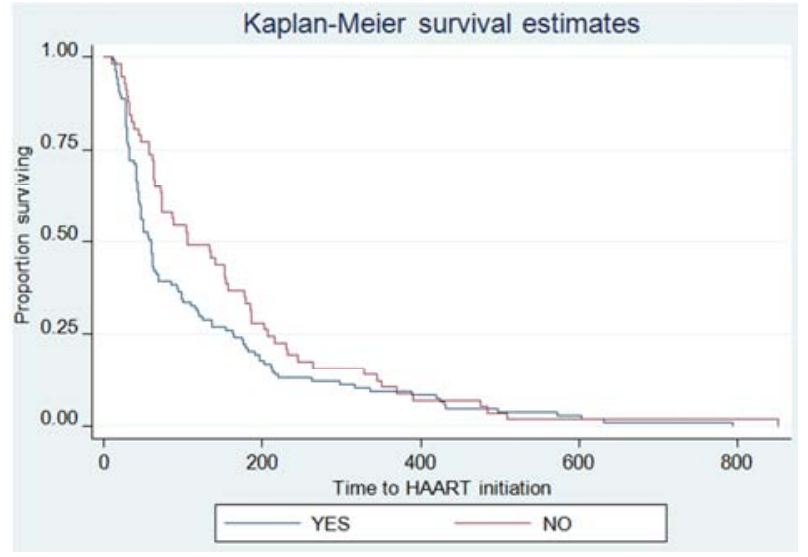

Figure 8. Kaplan Meier survival estimates for knowledge of diagnosed $H I V+$ family member.

In this plot, it was observable that knowledge of diagnosed
$\mathrm{HIV}+$ family member produced observable difference in the plots showing time to HAART eligibility event. This observation was then to be put to a statistical test to show if indeed this was to be confirmed. The log rank test was run to confirm these.

Table 6. Log rank for knowledge of diagnosed HIV+ family member.

\begin{tabular}{llll}
\hline $\begin{array}{l}\text { knowledge of diagnosed } \\
\text { HIV+ family member }\end{array}$ & $\begin{array}{l}\text { Events } \\
\text { observed }\end{array}$ & $\begin{array}{l}\text { Events } \\
\text { expected }\end{array}$ & Rank \\
\hline YES & 107 & 95.7 & 1545 \\
NO & 57 & 63.3 & -1545 \\
Total & 164 & 164 & \\
\hline
\end{tabular}

Chi2 $(1)=6.80$ p-value $=0.0091$

This was statistically significant and would be included in the final model.

\subsection{Cox Proportional for Continuous Variable}

The data had 2 continuous variables taken at enrollment

\subsubsection{Cox Proportional Hazard for Age at Enrollment}

Cox regression was carried out with the ties handled by the Breslow method for ties.

Table 7. Cox regression output for age.

\begin{tabular}{|c|c|c|c|c|c|}
\hline$-^{\mathbf{t}}$ & $\begin{array}{l}\text { Hazard } \\
\text { ratio }\end{array}$ & $\begin{array}{l}\text { Standard } \\
\text { error }\end{array}$ & $\mathbf{Z}$ & $\mathbf{P}>\mathbf{I z I}$ & $\begin{array}{l}\text { [95\%Confidence } \\
\text { Interval }\end{array}$ \\
\hline Age & 1.0195515 & .0067639 & 2.91 & 0.004 & 1.006344 \\
\hline
\end{tabular}

Age at enrollment was considered for the final model since the p-value was 0.004 and was therefore statistically significant in influencing time to HAART initiation

\subsubsection{Cox Proportional Hazard for BMI at Enrollment}

Cox regression was carried out with the ties handled by the Breslow method for ties.

Table 8. Cox regression output for BMI.

\begin{tabular}{|c|c|c|c|c|c|}
\hline$-^{\mathbf{t}}$ & $\begin{array}{l}\text { Hazard } \\
\text { ratio }\end{array}$ & $\begin{array}{l}\text { Standard } \\
\text { error }\end{array}$ & $\mathbf{Z}$ & $\mathbf{P}>\mathbf{I z I}$ & $\begin{array}{l}\text { [95\%Confidence } \\
\text { Interval }\end{array}$ \\
\hline BMI & 0.9991903 & .001323 & $\begin{array}{l}- \\
0.61\end{array}$ & 0.542 & 0.9965922 \\
\hline
\end{tabular}

\subsubsection{Model Building}

The variables found to be significant were considered for the final model. These were gender, who stage at enrollment, knowledge of a family member with known HIV+ status and the age at enrollment.

\subsubsection{Cox Proportional Hazard with All Significant Covariates}

The four variables found to be significant were fit in to a cox PH model and the findings were as below:

Table 9. Cox regression output for significant variables.

\begin{tabular}{llllll}
\hline $\mathbf{t}$ & Hazard ratio & Standard error & Z & P>IzI & [95\%Confidence Interval \\
\hline Gender & 0.8903265 & 0.1476927 & -0.70 & 0.484 & 0.6432012 \\
Enrollment Age & 1.016259 & 0.0068615 & 2.39 & 0.017 & 1.2324 \\
WHO stage at enrolment & 1.696663 & 0.2727343 & 3.29 & 0.001 & 1.002899 \\
Knowledge of HIV+ family member & 0.79708676 & 0.133139 & -1.36 & 0.175 & 05745496 \\
\hline
\end{tabular}


After analysis, the p-values for gender and knowledge of a family member with known HIV+ status were found to be non-significant 0.484 and 0.175 respectively and were eliminated in final model.

\subsubsection{Cox Proportional Hazard Fit Model}

After the first fitting the statistically significant covariates were incorporated in the final fit model and the findings were as below:

Table 10. Cox regression output for the final model.

\begin{tabular}{llllll}
\hline $\mathbf{t}$ & Hazard ratio & Standard error & $\mathbf{z}$ & P>IzI & [95\%Confidence Interval] \\
\hline Age at enrollment & 1.01798 & 0.0065841 & 2.76 & 0.006 & 1.005157 \\
WHO stage at enrolment & 1.743588 & 0.27763384 & 3.49 & 1.030966 & 0.000 \\
\hline
\end{tabular}

\section{Discussions}

The study was nectitated by the need for early diagnosis of HIV. The assumption that patients will be diagnosed early in the course of HIV infection is crucial to the earlier initiation of ART. However, HIV infected patients are still diagnosed at later stages of disease. Despite the recommendations for routine, opt-out HIV screening in the health care setting regardless of perceptions about a patient's risk of infection [11] and the gradual increase in CD4 counts at first presentation to care, the median CD4 count of newly diagnosed patients remains below 350 cells/mm [12, 13]. Diagnosis of HIV infection delays differ significantly among different groups in a population $[14,15,16]$. The findings of this study showed that age of enrolment was a significant determinant of the time to HAART initiation on enrolment to care and treatment. The hazard ratio associated with age was 1.01798 (p-value 0.006) which means that with every unit increase in age the increase in the rate of time to HAART initiation as per national guidelines is $1.8 \%$. This may appear small but the public health burden of HIV-AIDS is large and the knowledge that older people have weak immunities and therefore suffer a higher rate of immune damage than corresponding younger ones should inform decision making. The results of this study were in agreement with the increasing risk of higher rates of disease progression with increase in age [17]. This raises the issue of whether or not older patients should be treated with a higher CD4+ cell counts a factor this study seeks to find out, especially in resource limited settings as is the case in Bondo.

The effect of marital status affection their progression to HAART initiation was found to be insignificant ( $p$-value 0.338 ) and did not contribute to the final model. This can be explained by the fact that there have been a lot of behavior change initiatives aiming at dealing with stigma and formation of support groups that have changed population perceptions. The study found significant difference in the time to HAART initiation between the male and female with the men having a higher rate of disease progression ( $p$-value 0.0275) to needing HAART management. This was indifferent from the Previous laboratory other studies by Anastos et al, 2000 which found that the mean HIV-RNA to vary between men and women [17] for a given CD4+ cell count strata, after adjustment for differences in measurement method, baseline cd4 counts, age, and clinical symptoms, HIV-1RNA levels were $32 \%$ to $50 \%$ lower in women than men at cd4 + counts more than 200 cells $/ \mathrm{mm} 3(\mathrm{p}<.001)$. The effect of this findings were however not evident in our study that looked at the time to HAART as a dependent variable on gender. This could be explained by the fact that the sample had significantly more female than males and the better health seeking behavior of females in the catchment area.

The cox proportional hazard model with body mass index (BMI) was an insignificant fit (p-value-0.542) and as not included into the final model fit. It is a measure of nutritional status and its relationship to survival in HIV infection is important since a more than $10 \%$ involuntary weight loss in conjunction with chronic diarrhea and weakness is considered an AIDS defining illness as per CDC classification [18]. This could be explained by the fact that our inclusion criteria only captured WHO stage I and II who generally have more manifestation of involuntary weight loss with chronic diarrhea.

The entry point into a HIV-AIDS care provision center was found to be of no significance ( $p$-value 0.7716 ) to the time taken by enrolled patients to be eligible to HAART. The relationship between this entry point and prognosis of the disease in the patient was established to be not relating in the study. Newly enrolled patient having prior knowledge of a known family member who has been diagnosed as being $\mathrm{HIV}+$ had longer time to HAART eligibility (p-value 0.0091) compared to those who had no known family member. This though significant was found to be insignificant in the final cox proportional model. This was in agreement to the findings of some studies by Winchester et al, 2003 that HIVinfected patients make strategic decision on whom to disclose to their status disclosure is mainly to those physically and socially closest to them [19], this protects them from inadvertent disclosures by decreasing visible symptoms that make others aware of their symptoms.

The WHO stage of enrollment was found to be a significant determinant ( $p$-value 0.0000 ) of the time to HAART eligibility with a significance. The patient enrolled with WHO stage II had 74\% higher chance of faster progression to eligibility to HAART compared to those who were enrolled as WHO stage II in the final model fit. Therefore, to ensure that the current treatment guidelines have maximum impact, routine HIV screening per current Centers for Disease Control and Prevention and the WHO recommendations are essential.

Study limitations

During the study period, it was made aware that the ministry of health had implemented a Rapid Response 
Initiative (RRI) to increase the number of patients on HAART and this affected the true scenario as envisaged in the normal program undertakings. This explained the lack of patients for censoring at end of study and this affected the study findings.

Survival analysis requires reliable sources of data obtained from prospective cohort studies while we perform a retrospective design and use data recorded at the care site. The quality and accuracy of the estimations and associations primarily depended on the quality of the recorded data, whereas we were unable to verify the accuracy of the data. This issue may raise possibility of information bias.

\section{Conclusion and Recommendations}

In conclusion, the main factors found to affect the time to HAART initiation for the patients enrolled at Bondo Subcounty hospital in the study period were age of enrollment and the WHO stage of enrolment. Using Kaplan Meier and Cox regression the study was able to come up with several conclusions. First, the survival time to HAART eligibility was determined and risk factor of age of enrollment and patients WHO stage at enrolment were the main risk factors affecting progression to drug eligibility. Secondly, Age of enrolment and WHO stage of enrollment were the main variables captured in $\mathrm{MOH} 257$ that inform on hiv-haart naïve disease progression to the haart requiring end point. Lastly, the null hypothesis was rejected.

The study recommends the Bondo Sub-County Hospital for the complete filing up of $\mathrm{MOH} 257$ which enabled the extraction of the relevant data for analysis. During the study period, it was made aware that the Ministry of Health had implemented a Rapid Response Initiative (RRI) to increase the number of patients on HAART and this affected the true scenario as envisaged in the normal program undertakings. This explained the lack of patients for censoring at end of study and this affected the study findings. Survival analysis requires reliable sources of data obtained from prospective cohort studies while a retrospective design was performed and used data recorded at the care site. The quality and accuracy of the estimations and associations primarily depended on the quality of the recorded data, whereas it was not possible to verify the accuracy of the data. This issue may raise possibility of information bias. This study therefore needs to be done in a prospective manner, incorporating time dependent covariates so as to give a clear picture of the other covariates not picked in this study.

\section{Acknowledgements}

I would like to express my special thanks to the staff at Bondo Sub County Hospital headed by Isaac Ng'iendo. Dr. Hilary Kipruto and Charlotte Nyaboe for your assistance and input throughout the project cycle. God bless you

\section{References}

[1] National Aids Control Council Kenya(NACC). (2014). KENYA HIV COUNTY PROFILES. NAIROBI: National Aids Control Council Kenya.

[2] WHO. (2014). GLOBAL UPDATE ON HEALTH SECTOR RESPONSE TO HIV. GENEVA: WHO.

[3] Kenya National Bearau of Statistic(KNBS). (2013). statistical abstract 2013. Nairobi: KNBS.

[4] National AIDS/STI Control Program(NASCOP). (2011). Guidelines for aantiretroviral therapy in Kenya. Nairobi: NASCOP.

[5] National Aids Control Council kenya(NACC). (2014). Kenya aids progressive report. nairobi: natinal aids contol council.

[6] NASCOP. (2014). guidelines on use of ntiretroviral drugs for treating and preventing HIV infection:a rapid advise. NAIROBI: MINISTRY OF HEALTH.

[7] Kleinbaum, D. G., \& Klein, M. (2005). Survival analysis:a self learning text. New York: springer.

[8] Lee, E., \& wang, J. W. (2003). Statistical Methods for Survival Data Analysis. New Jersey: John Wiley \& Sons.

[9] Kaplan, E. L., \& Meier, P. (1958). Non-parametric Estimation from Incomplete Observation. journal of American Statistical Association, 457-48.

[10] Mantel, N., \& Haenszel, W. (1959). Statistical Aspect of the Analysis of Data from Retrospetive Studies od Diseases. Journal of National Cancer Institute, 719-748.

[11] Branson BM, Handsfield HH, Lampe MA, et al. Revised recommendations for HIV testing of adults, adolescents, and pregnant women in health-care settings. MMWR Recomm Rep. Sep $22 \quad 2006 ; 55(\mathrm{RR}-14): 1-17$. Available at http://www.ncbi.nlm.nih.gov/entrez/query.fcgi?cmd=Retrieve $\& \mathrm{db}=$ PubMed\&dopt=Citation\&list_uids=16988643.

[12] Granich RM, Gilks CF, Dye C, De Cock KM, Williams BG. Universal voluntary HIV testing with immediate antiretroviral therapy as a strategy for elimination of HIV transmission: a mathematical model. Lancet. Jan 3 2009;373(9657):48-57. Available at

http://www.ncbi.nlm.nih.gov/entrez/query.fcgi?cmd=Retrieve $\& \mathrm{db}=$ PubMed\&dopt=Citation\&list_uids=19038438.

[13] Althoff KN, Gange SJ, Klein MB, et al. Late presentation for human immunodeficiency virus care in the United States and Canada. Clin Infect Dis. Jun 1 2010;50(11):1512-1520. Available at http://www.ncbi.nlm.nih.gov/pubmed/20415573.

[14] Wolbers M, Bucher HC, Furrer H, et al. Delayed diagnosis of HIV infection and late initiation of antiretroviral therapy in the Swiss HIV Cohort Study. HIV Med. Jul 2008;9(6):397-405. Available http://www.ncbi.nlm.nih.gov/entrez/query.fcgi?cmd=Retrieve $\& \mathrm{db}=$ PubMed\&dopt=Citation\&list_uids $=18410354$.

[15] Centers for Disease Control and Prevention. Late HIV testing - 34 states, 1996-2005. MMWR Morb Mortal Wkly Rep. Jun 26 2009;58(24):661-665. Available at http://www.ncbi.nlm.nih.gov/entrez/query.fcgi?cmd=Retrieve $\& \mathrm{db}=$ PubMed\&dopt $=$ Citation\&list_uids=19553901. 
[16] Grigoryan A, Hall HI, Durant T, Wei X. Late HIV diagnosis and determinants of progression to AIDS or death after HIV diagnosis among injection drug users, 33 US States, 19962004. PLoS One. 2009;4(2):e4445. Available at http://www.ncbi.nlm.nih.gov/entrez/query.fcgi?cmd=Retrieve $\& \mathrm{db}=$ PubMed\&dopt=Citation\&list_uids=19214229.

[17] Anastos, k., Gange, S. J., Lau, B., Weiser, B., \& Detels, R. (2000). Association of race and gender with HIV-1 RNA levels and immunologic progression. journal of acquired immunodeficiency syndrome, 218-226.
[18] Faauci, A. S., \& Lane, H. C. (2005). Harrison's Principles of Internal Medicine 16 Edition (Edited by: Fauci AS, Lane HC). McGraw-Hill 2005, 1:p1076-1139. McGraw-Hill 2005, 1:p1076-1139.

[19] Winchester, M. S., McGrath, J. W., Kaawa-mafigiri, D., Namutiiba, F., Ssendegye, G., Nalwoga, A., et al. (2013). Early HIV disclosure and nondisclosure among men and women on antiretroviral treatment in Uganda. AIDS Care: Psychological and Socio-medical Aspects of AIDS/HIV, 12531258. 